University of South Carolina

Scholar Commons

4-17-2007

\title{
Computation of Correlation Functions and Wave Function Projections in the Context of Quantum Trajectory Dynamics
}

\author{
Sophya Garashchuk \\ University of South Carolina--Columbia, sgarashc@chem.sc.edu
}

Follow this and additional works at: https://scholarcommons.sc.edu/chem_facpub

Part of the Chemistry Commons

\begin{abstract}
Publication Info
Published in Journal of Chemical Physics, Volume 126, Issue 15, 2007, pages 154104-.

(C) Journal of Chemical Physics 2007, American Institute of Physics.
\end{abstract}

This Article is brought to you by the Chemistry and Biochemistry, Department of at Scholar Commons. It has been accepted for inclusion in Faculty Publications by an authorized administrator of Scholar Commons. For more information, please contact digres@mailbox.sc.edu. 


\title{
Computation of correlation functions and wave function projections in the context of quantum trajectory dynamics
}

\author{
Sophya Garashchuk \\ Department of Chemistry, University of South Carolina, Columbia, South Carolina 29223
}

(Received 20 December 2006; accepted 26 February 2007; published online 17 April 2007)

\begin{abstract}
The de Broglie-Bohm formulation of the Schrödinger equation implies conservation of the wave function probability density associated with each quantum trajectory in closed systems. This conservation property greatly simplifies numerical implementations of the quantum trajectory dynamics and increases its accuracy. The reconstruction of a wave function, however, becomes expensive or inaccurate as it requires fitting or interpolation procedures. In this paper we present a method of computing wave packet correlation functions and wave function projections, which typically contain all the desired information about dynamics, without the full knowledge of the wave function by making quadratic expansions of the wave function phase and amplitude near each trajectory similar to expansions used in semiclassical methods. Computation of the quantities of interest in this procedure is linear with respect to the number of trajectories. The introduced approximations are consistent with approximate quantum potential dynamics method. The projection technique is applied to model chemical systems and to the $\mathrm{H}+\mathrm{H}_{2}$ exchange reaction in three dimensions. () 2007 American Institute of Physics. [DOI: 10.1063/1.2717931]
\end{abstract}

\section{INTRODUCTION}

Development of rigorous semiclassical dynamics methods for molecular systems, combining favorable scaling of classical mechanics with respect to system size with an accurate quantum-mechanical $(\mathrm{QM})$ description of molecular motion, is a long-standing goal of theoretical chemistry. The de Broglie-Bohm ${ }^{1}$ formulation of the time-dependent Schrödinger equation (TDSE) is conceptually appealing as a foundation for such semiclassical method, because it can, in principle, exactly describe QM effects, yet it is formulated in terms of trajectories. This may lead to a seamless quantum/ classical or semiclassical/classical description of large molecular systems with quantum effects included in selected degrees of freedom.

Let us consider the basic one-dimensional TDSE describing a particle of mass $m$ moving under the influence of a potential $V$. Writing the wave function in a polar form

$$
\psi(x, t)=A(x, t) e^{i S(x, t) / \hbar}=\sqrt{\rho(x, t)} e^{i S(x, t) / \hbar},
$$

in terms of real functions $A(x, t)$ and $S(x, t)$, and using probability density

$$
w(x, t)=\rho(x, t) d x(t),
$$

TDSE can be recast as

$$
\begin{aligned}
& \frac{d S(x, t)}{d t}=\frac{p^{2}(x, t)}{2 m}-(V+U), \\
& \frac{d w(x, t)}{d t}=0 .
\end{aligned}
$$

$U$ is the quantum potential,

$$
U=-\frac{\hbar^{2}}{2 m}\left(r^{2}(x, t)+r^{\prime}(x, t)\right),
$$

and $p(x, t)$ and $r(x, t)$ are the classical and nonclassical components of the momentum operator, respectively,

$$
\begin{aligned}
& p(x, t)=S^{\prime}(x, t), \\
& r(x, t)=\frac{A^{\prime}(x, t)}{A(x, t)} .
\end{aligned}
$$

Prime denotes differentiation with respect to coordinate. Equations (3) and (4) imply that $\psi(x, t)$ can be defined in terms of trajectories evolving according to the Hamilton equations of motion in the presence of the combined potential, $V+U$. The wave function density, $\rho(x, t)$, within the volume element $d x(t)$ associated with each trajectory defined by Eq. (2)—it will be referred to as the trajectory weight-is constant in time. ${ }^{2}$ This property is explicitly used in the approximate quantum potential (AQP) method ${ }^{2,3}$ in contrast to other implementations of the de Broglie-Bohm formulation where the wave function density is found from differential equations. ${ }^{4,5}$ In the AQP method for a numerical implementation the initial wave function is discretized in a set of trajectories with initial positions $\left\{x_{i}\right\}$, momenta $\left\{p_{i}=S^{\prime}\left(x_{i}\right)\right\}$, and weights $w_{i}=\rho\left(x_{i}, 0\right) d x_{i}(0)$. All quantum effects enter the quantum trajectory formulation through a nonlocal and, in general, singular quantum potential $U$, which makes exact implementation of the quantum trajectory formulation very challenging. ${ }^{6-9}$ In the semiclassical limit, $\hbar \rightarrow 0$, for nonzero densities the quantum potential vanishes, $U \rightarrow 0$. (Atomic units $\hbar=1$ are used below.)

We have implemented this formulation semiclassically defining $U$ in terms of approximate $r(x, t) .{ }^{3}$ The nonclassical momentum $r(x, t)$ is expanded in a small basis, and the ex- 
pansion coefficients are found variationally yielding the energy-conserving dynamics with AQP. Linear basis functions give exact dynamics of Gaussian wave functions. Working with trajectory weights rather than finding timedependent wave function density is a major simplification that gives linear scaling with the number of trajectories to the AQP approach and improves its accuracy. Moreover, the expectation value evaluation of position-dependent operators is straightforward. For an operator $\hat{O}$,

$$
\langle\hat{O}(t)\rangle=\int O(x) \rho(x, t) d x=\sum_{i} O\left(x_{i}(t)\right) w_{i}
$$

where index $i$ labels trajectories.

In the context of nuclear dynamics, however, one often needs to analyze correlation functions of the type

$$
C(t)=\langle\phi(0) \mid \psi(t)\rangle=\int \phi^{*}(x, 0) \psi(x, t) d x,
$$

and wave function projections that cannot be expressed as single sums over trajectories. The integrand in Eq. (9) does not include $\rho(x, t)=|\psi(x, t)|^{2}$, which allows one to use trajectory weights as in Eq. (8). As discussed in Ref. 3, $\psi(x, t)$ can be reconstructed through a convolution procedure centered on $x$ which requires summation over trajectories for each $x$. This approach is quite accurate, but expensive since, if used, Eq. (9) would entail a double summation over the trajectories. It is most useful if the wave function needs to be analyzed just a few times in the course of dynamics. If $C(t)$ is needed on a time interval, we can use a global fit of $\psi(x, t)$ to a simple function (for instance, to a Gaussian) on a subspace of non-negligible $|\phi(x, 0)|$. Computation of the fitting parameters can be made linear with respect to the number of trajectories. The accuracy of integration is determined by the accuracy of the wave function amplitude evaluation.

In the remainder of the article we describe how to compute correlation functions and wave function projections in an efficient manner, assuming Gaussian shape of $\psi(x, t)$ around each trajectory, which is more accurate than the global fit, but with the linear scaling with respect to the number of trajectories. The method is based on the Wigner transformation $^{10}$ in double integrals over the coordinate space. After the transformation integration with respect to the sum of coordinates, $x$, is performed exactly. The integral with respect to the difference of coordinates, $y$, is performed analytically by expanding the exponent of the integrand through the second order in $y$. This approximation used, for example, to combine two semiclassical propagators in Ref. 11, is accurate in the semiclassical limit when oscillatory integrals are dominated by the contributions from the lowest terms.

The approach described below can be used with any trajectory-based propagation method which conserves probability density. Numerical illustrations are given for oneand two-dimensional model chemical systems and for the $\mathrm{H}+\mathrm{H}_{2}$ reaction described in the Jacobi coordinates.

\section{WAVE FUNCTION OVERLAPS FROM LOCAL QUADRATIC EXPANSIONS}

\section{A. Wave packet correlation functions}

In nuclear dynamics studies wave packet correlation functions are used, in particular, to compute photodissociation spectra and energy-resolved reaction probabilities. As discussed in the Introduction, the AQP method uses conservation of density probabilities given by Eq. (4) bypassing computation of density $\rho(x, t)$. Therefore, accurate reconstruction of $\psi(x, t)$ from the convolution procedure leads to a double summation over the trajectories in the wave packet correlation function,

$$
C(t)=\int \phi^{*}(x, 0) A(x, t) e^{i S(x, t)} d x .
$$

As an exception, an autocorrelation function of an initially real wave packet $\psi(x, 0)$ is expressed ${ }^{12}$ simply as

$$
C(2 t)=\left\langle\left(e^{-i H t} \psi(0)\right)^{*} \mid e^{-i H t} \psi(0)\right\rangle=\sum_{i} e^{2 i S\left(x_{i}, t\right)} w_{i} .
$$

To obtain $C(t)$ from a single summation over the trajectories for arbitrary wave functions, it is necessary to have in the integrand the wave function density $\rho(x, t)$, not the amplitude $A(x, t)$ as in Eq. (10). We can compute

$$
C^{2}(t)=\iint \phi^{*}\left(x_{1}, 0\right) \psi\left(x_{1}, t\right) \psi\left(x_{2}, t\right) \phi^{*}\left(x_{2}, 0\right) d x_{1} d x_{2}
$$

and make a change of variables as in the Wigner transformation, ${ }^{10}$

$$
x=\frac{\left(x_{1}+x_{2}\right)}{2}, \quad y=x_{2}-x_{1} .
$$

Expressing the old coordinates $\left(x_{1}, x_{2}\right)$ in terms of the new coordinates $(x, y)$ we have

$$
\begin{aligned}
C^{2}(t)= & \iint \phi^{*}\left(x-\frac{y}{2}, 0\right) \psi\left(x-\frac{y}{2}, t\right) \\
& \times \psi\left(x+\frac{y}{2}, t\right) \phi^{*}\left(x+\frac{y}{2}, 0\right) d x d y .
\end{aligned}
$$

Representing $\psi(x, t)$ in a polar form through its density $\rho(x, t)$ and phase $S(x, t)$, and making the Taylor expansion of the exponent through the third order in $y$, Eq. (14) becomes

$$
\begin{aligned}
C^{2}(t) \approx & \iint \rho(x, t) e^{2 i S(x, t)+\left(r^{\prime}(x, t)+i p^{\prime}(x, t)\right) y^{2} / 4} \\
& \times \phi^{*}\left(x-\frac{y}{2}, 0\right) \phi^{*}\left(x+\frac{y}{2}, 0\right) d x d y .
\end{aligned}
$$

In the expansion above odd powers of $y$ cancel by symmetry. Integration over $y$ is trivial for a Gaussian stationary wave packet $\phi(x, 0)$,

$$
\phi(x, 0)=\left(\frac{2 \alpha}{\pi}\right)^{1 / 4} \exp \left(-\alpha\left(x-x_{0}\right)^{2}+i p_{0}\left(x-x_{0}\right)\right),
$$

giving 


$$
C^{2}(t) \approx \sum_{i} \sqrt{\frac{2 \pi}{\beta_{i}}}\left(\phi^{*}\left(x_{i}, t\right)\right)^{2} e^{2 i S\left(x_{i}, t\right)} w_{i} .
$$

Parameter $\beta_{i}$ is, in general, trajectory dependent,

$$
\beta_{i}=\alpha-\frac{1}{2}\left(r^{\prime}\left(x_{i}, t\right)+i p^{\prime}\left(x_{i}, t\right)\right) .
$$

Exact nonclassical momentum $r(x, t)$ is not computed in the AQP method, but we can use its approximate form available from the AQP dynamics. Classical momentum $p(x, t)$ is known along the trajectories and it can be represented in the same basis as $r(x, t)$ so that the derivatives in Eq. (18) are approximated on the same level with analytical functions. Using $|\phi(x, 0)|^{2}$ as a weighting function in the computation of the expansion coefficients will give more accurate $\beta_{i}$. Expansions for $r(x, t)$ and $p(x, t)$ in a linear basis are exact for a Gaussian wave packet evolving in a locally quadratic potential. Taking the square root of Eq. (17) ensuring its continuity in time gives complex $C(t)$.

Gaussian form of $\phi(x, 0)$ is a typical choice for the analysis of outgoing wave function components in scattering problems, but representation of an arbitrary $\phi(x, 0)$ in the Gauss-Hermite basis ${ }^{13}$ will allow analytical integration over $y$ for non-Gaussian $\phi(x, 0)$ as well (analogous to the derivation in Sec. II B).

A more general type of correlation functions,

$$
\langle\hat{B}(t) \hat{B}\rangle=\left\langle\psi(0)\left|e^{i H t} \hat{B} e^{-i H t} \hat{B}\right| \psi(0)\right\rangle,
$$

where $\hat{B}$ is an operator, is discussed in the context of trajectory dynamics in Ref. 14. Note that despite the presence of two evolution operators one cannot extend the above derivation and use the probability density conservation in Eq. (19). Unlike Eq. (12) in Eq. (19) the evolution operators act on different bra and ket wave functions (unless $\hat{B}$ is an identity operator),

$$
\langle\hat{B}(t) \hat{B}\rangle=\langle\psi(t)|\hat{B}| \phi(t)\rangle, \quad \phi(0)=\hat{B} \psi(0) .
$$

\section{B. Projection onto a vibrational basis}

In order to project a wave function $\psi(x, t)$ onto a real basis $\left\{\chi_{n}\right\}$ needed, for example, in the vibrational distribution analysis in scattering, one has to compute overlap integrals

$$
c_{n}=\left\langle\chi_{n} \mid \psi\right\rangle .
$$

(We consider projection at a given time $t$ and suppress the time variable in the argument throughout this subsection.) Expression involving a single summation over trajectories can be obtained by computing $\left|c_{n}\right|^{2}$ or, more generally, elements $t_{n m}$ of a matrix $\mathbf{T}$,

$$
t_{n m}=c_{n}^{*} c_{m}
$$

and making quadratic expansions in the exponent of $\psi(x)$ around each trajectory. Let us project onto the GaussHermite vibrational basis $\left\{\chi_{n}\right\}$,

$$
\begin{aligned}
t_{n m} & =\left\langle\psi \mid \chi_{n}\right\rangle\left\langle\chi_{m} \mid \psi\right\rangle \\
& =\iint \psi^{*}\left(x_{1}\right) \chi_{n}\left(x_{1}\right) \chi_{m}\left(x_{2}\right) \psi\left(x_{2}\right) d x_{1} d x_{2},
\end{aligned}
$$

make change of variables given by Eq. (13), and expand the exponent of $\psi$ through the second order in $y$. Note that this expansion is different from the semiclassical $\hbar$ expansions of the Wigner functions. ${ }^{15}$ The result is somewhat different from Eq. (15) because of conjugation in the definition of $t_{n m}$ :

$$
t_{n m} \approx \int \rho(x) \omega_{n m}(x) d x,
$$

where

$$
\omega_{n m}(x)=\int \exp \left(\frac{r^{\prime}}{4} y^{2}+i p y\right) \chi_{n}\left(x-\frac{y}{2}\right) \chi_{m}\left(x+\frac{y}{2}\right) d y .
$$

Functions $r^{\prime}$ and $p$, in general, depend on $x$, i.e., on the trajectory index. If $\psi(x)$ is sufficiently delocalized compared to $\chi_{n}(x)$ so that $r^{\prime}$ can be neglected with, then $\omega_{n m}(x)$ given by Eq. (25) is proportional to the Wigner transform of the harmonic oscillator eigenstates. (The transforms including the nondiagonal elements are given in Appendix A.) Otherwise, for the Gauss-Hermite functions,

$$
\chi_{n}=\sqrt{\frac{k}{2^{n} n ! \pi^{1 / 2}}} H_{n}(k x) \exp \left(-\frac{k^{2} x^{2}}{2}\right),
$$

denoting $r^{\prime}=-a$ we obtain

$$
\omega_{n m}(x)=\sum_{i=0}^{n} \sum_{j=0}^{m} C_{i}^{n} C_{j}^{m} f_{0} H_{i+j}(\sqrt{2} k x) H_{m+n-i-j}(\sqrt{2} \kappa p) .
$$

$C_{i}^{n}$ are the binomial coefficients. Parameter $\kappa$ is defined as

$$
\begin{aligned}
& \kappa=\frac{k}{\sqrt{k^{4}-a^{2}}} \text { for } k^{2}>a, \\
& \kappa=\frac{i k}{\sqrt{\left|k^{4}-a^{2}\right|}} \text { for } k^{2}<a,
\end{aligned}
$$

and $f_{0}$ is

$$
\begin{aligned}
f_{0}= & \frac{2 k}{\sqrt{k^{2}+a}} \frac{2^{-n-m}}{\sqrt{n ! m !}} \sqrt{\frac{a-k^{2}}{a+k^{2}}}{ }^{n+m-i-j}(-1)^{n-i} \\
& \times \exp \left(-k^{2} x^{2}-\frac{p^{2}}{k^{2}+a}\right) .
\end{aligned}
$$

For a special case of $k^{2}=a$, we have

$$
\begin{aligned}
& \omega_{n m}(x)=\sum_{i=0}^{n} \sum_{j=0}^{m} C_{i}^{n} C_{j}^{m} f_{0} H_{i+j}(\sqrt{2} k x)\left(\frac{-i p}{k}\right)^{n+m-i-j}, \\
& f_{0}=(-1)^{n-i} \frac{2^{-(i+j+n+m-1) / 2}}{\sqrt{n ! m !}} \exp \left(-k^{2} x^{2}-\frac{p^{2}}{2 k^{2}}\right) .
\end{aligned}
$$

In terms of discrete trajectories the matrix elements given by Eq. (24) are 


$$
t_{n m} \approx \sum_{l} \omega_{n m}\left(x_{l}\right) w_{l}
$$

Momentum $p$ is a classical momentum known for each trajectory and $r^{\prime}=r^{\prime}\left(x_{l}\right)$ can be approximated as outlined in Sec. II A. In practice we recursively compute the Hermite polynomials of $x_{l}$ and $p_{l}$ up to the order of $2 N$ ( $N$ is the basis size) and then carry out summation according to Eq. (27).

To obtain the probabilities of a system to be in a particular vibrational eigenstate $\widetilde{\chi}_{n}$, the vibrational eigenstates can be expanded in the Gauss-Hermite basis, $\tilde{\boldsymbol{\chi}}=\mathbf{M} \boldsymbol{\chi}$, where $\mathbf{M}$ is the transformation matrix. The probabilities $\tilde{t}_{n n}=\left|\left\langle\tilde{\chi_{n}} \mid \psi\right\rangle\right|^{2}$ are given by the diagonal elements of the matrix $\widetilde{\mathbf{T}}$,

$$
\widetilde{\mathbf{T}}=\mathbf{M T M}^{T} .
$$

\section{NUMERICAL EXAMPLES}

\section{A. Energy-resolved probabilities}

As an illustration we compute the energy-resolved transmission probabilities for the Eckart barrier mimicking the transition state of the $\mathrm{H}+\mathrm{H}_{2}$ reaction in one dimension. The correlation function formulation of the $S$ matrix ${ }^{16}$ is used. The system is described in Ref. 3. The correlation function $C(t)$ given by Eq. (9) is an overlap of a time-dependent reactant wave packet $\psi(x, t)$ and a stationary product wave packet $\phi(x, 0)$. Wave function $\phi(x, 0)$ is given by Eq. (16) with the parameter values $\alpha=6, x_{0}=3$, and $p_{0}=6$. Wave function $\psi(x, 0)$ is initially also a Gaussian,

$$
\psi(x, 0)=\left(\frac{2 \alpha}{\pi}\right)^{1 / 4} e^{-\alpha\left(x+x_{0}\right)^{2}+i p_{0}\left(x+x_{0}\right)} .
$$

Figure 1(a) compares the semiclassical $C(t)$ computed using Eq. (17) with the QM result obtained with the split-operator method. ${ }^{17}$ The semiclassical propagation $[C(t)$ shown with the dash] is implemented using global linear approximation to $r(x, t)$ resulting in the linear quantum force (LQF). Small phase lag of the semiclassical $C(t)$ at late times translates into the energy shift of the $S$ matrix shown on panel (b). The discrepancy between the exact and approximate correlation functions is due to the propagation error as verified by performing a more accurate semiclassical calculation (results marked as circles). In the second trajectory calculation we have used AQP obtained from $r(x, t)$ linearized on subspaces: seven Gaussian domains were defined as described in Ref. 18 - their centers are equally spaced from $q_{1}=-2.0$ to $q_{7}$ $=1.0$ and their width parameter is $\beta=5.0 a_{0}^{-2}$ - augmented by the domain complementing their sum to unity. Clearly, more accurate AQP nearly eliminates the discrepancy with the QM results.

\section{B. Vibrational projections}

In the second example we apply the projection formulation given by Eqs. (27) and (33) to the collinear model of the ICN dissociation. ${ }^{19,20}$ The initial wave function and the quantum trajectory implementation using LQF are described in Ref. 18. As ICN dissociates on the excited electronic potential energy surface we compute projections of the corre-
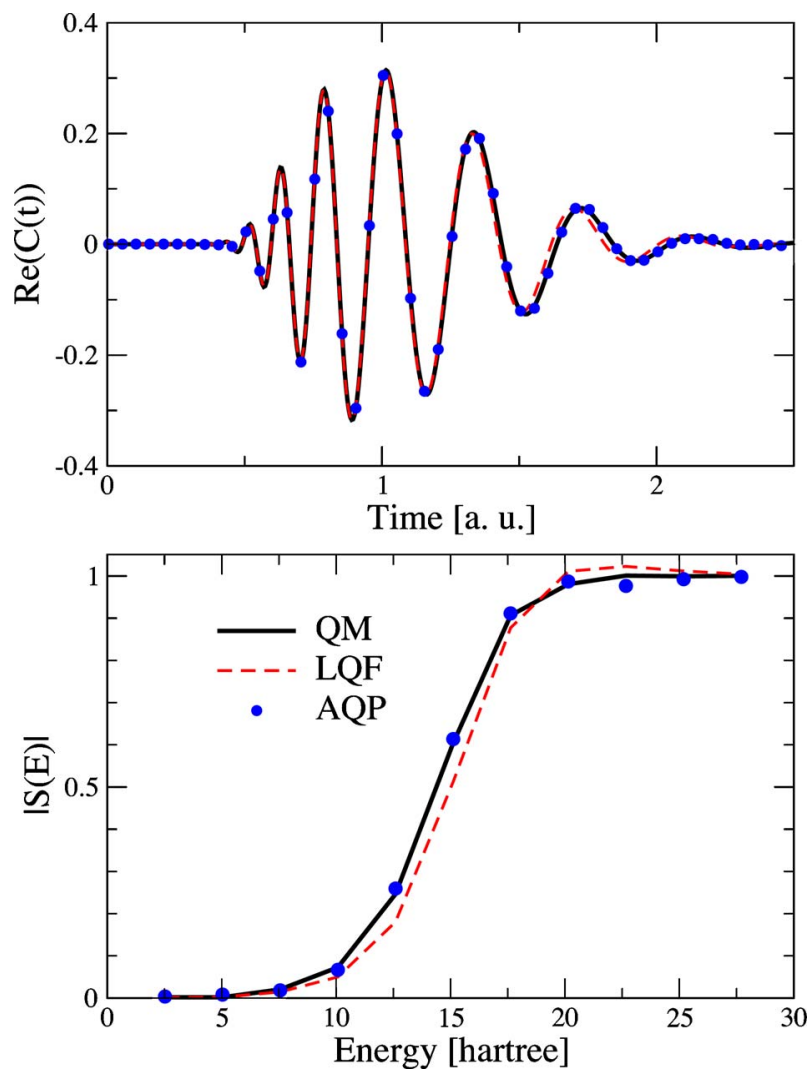

FIG. 1. (Color online) Scattering on the Eckart barrier: real part of the wave packet correlation function (top) and transmission coefficient, $|S(E)|$ (bottom). Results obtained with QM (solid line), LQF (dash), and AQP with subspaces (circles) methods are shown on both panels.

sponding $\psi(x, t)$ onto the eigenstates of $\mathrm{CN}$ which are the harmonic oscillator eigenfunctions in the given model.

Derivation in Sec. II B is given for one-dimensional wave function. In general, in more than one dimension we have to project $\psi$ onto an eigenstate specified by a complete set of quantum numbers. For scattering or dissociating systems the energy eigenstates in the asymptotic regions become direct products of eigenstates in the translational degree of freedom with outgoing asymptotic boundary condition and in the internal degrees of freedom. For the given problem these are outgoing free wave $\zeta_{E}$ associated with the translational energy $E$ in I-CN separation coordinate and the $\mathrm{CN}$ vibrational eigenfunction $\chi_{n}$. To find the vibrational projections one integrates the fully defined projections over the translational energy,

$$
\begin{aligned}
t_{n n} & =\int\left\langle\psi \mid \chi_{n} \times \zeta_{E}\right\rangle\left\langle\zeta_{E} \times \chi_{n} \mid \psi\right\rangle d E \\
& =\int\left|\int \psi(x, R) \chi_{n}(x) d x\right|^{2} d R .
\end{aligned}
$$

In Eq. (36) completeness of $\zeta_{E}$,

$$
\int \zeta_{E}^{*}\left(R_{1}\right) \zeta_{E}\left(R_{2}\right) d E=\delta\left(R_{1}-R_{2}\right)
$$

eliminates one of the integrals over the translational degree of freedom denoted as $R$. Therefore, Eq. (33) is valid in more than one degree of freedom if the vibrational motion is as- 


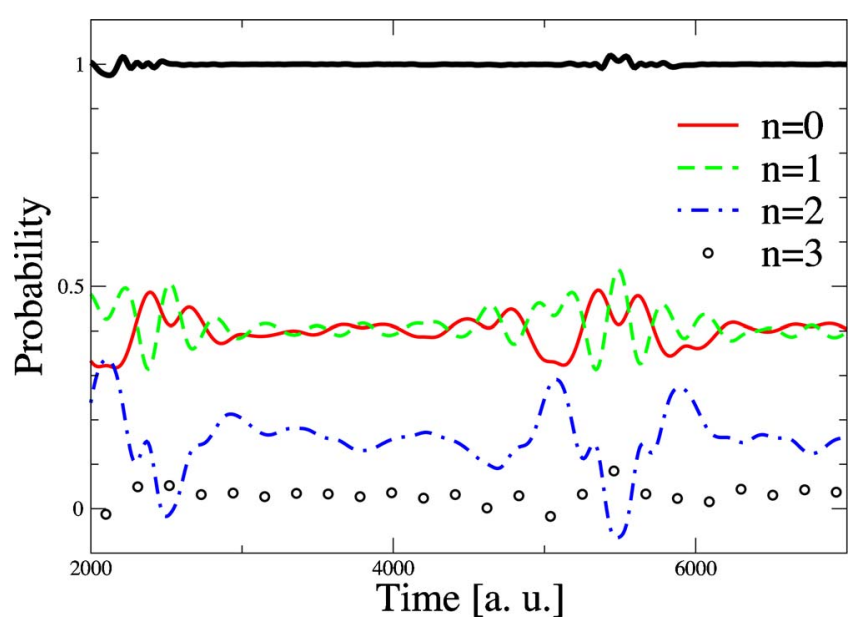

FIG. 2. (Color online) Wave function projections on to $\mathrm{CN}$ vibrational states.

ymptotically separable. In Eqs. (27)-(32) the coordinate $x$ now represents the vibrational degree of freedom. Functions $p$ and $r$ represent the $x$ components of the classical, $\mathbf{p}=\nabla S$, and nonclassical, $\mathbf{r}=A^{-1} \nabla A$, momenta of a trajectory and $a$ $=-\partial r / \partial x$. Integration over $R$ is included into summation over the trajectories. Computation of the QM vibrational projections in terms of correlation functions is given in Appendix B.

Projection coefficients $t_{n n}$ obtained with the AQP method are shown on Fig. 2 as functions of time. If the dynamics and projections were exact the coefficients would be constant once the wave packet moved into the asymptotic region. The semiclassical coefficients exhibit oscillations around $t$ $=5500$ a.u. The AQP propagation introduces some error, but the negative projection indicates that there is a projection error present as well. Examination of the trajectory distribution at $t=5500$ shows that at this time the wave packet is substantially non-Gaussian; therefore the error is most likely to come from the global linear approximation to $r$ in Eq. (25). The trajectory distributions are shown on Fig. 3 for $t$ $=5500$ a.u. as well as for $t=0$ and for $t=7000$ a.u. when the projections are nearly constant. Because of the errors present in the trajectory calculation we average the coefficients

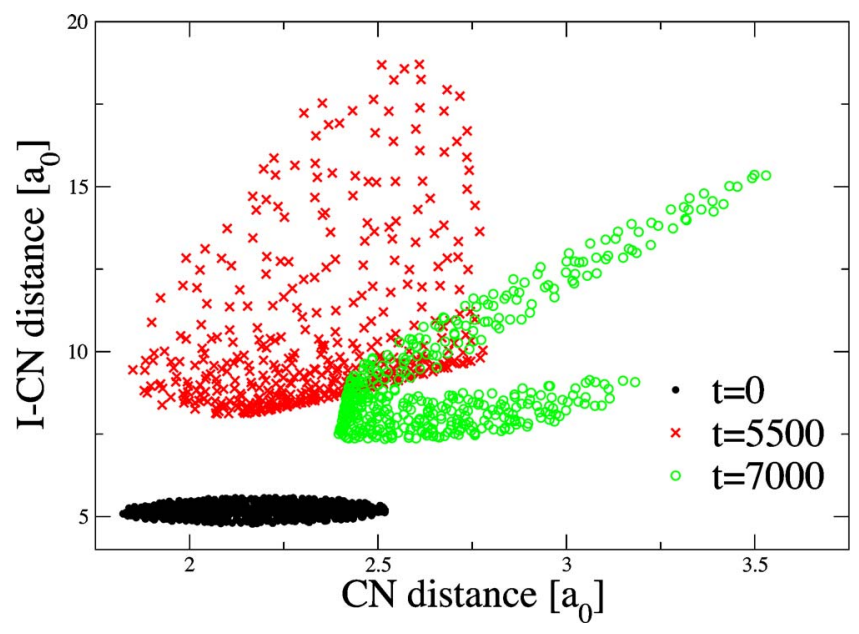

FIG. 3. (Color online) Trajectory positions during ICN dissociation.
TABLE I. Vibrational states distribution of $\mathrm{CN}$ in ICN dissociation obtained using QM and AQP propagations. The AQP wave function is projected as described in Sec. II B. $\sigma$ is the standard deviation due to averaging of the AQP probabilities over time.

\begin{tabular}{cccccc}
\hline \hline Vibrational state $n$ & 0 & 1 & 2 & 3 & 4 \\
\hline$P_{n} \mathrm{QM}$ & 0.402 & 0.403 & 0.162 & 0.031 & 0.003 \\
$P_{n} \mathrm{AQP}$ & 0.401 & 0.404 & 0.161 & 0.030 & 0.003 \\
$\sigma$ & 0.011 & 0.010 & 0.016 & 0.004 & 0.007 \\
\hline \hline
\end{tabular}

computed every 10 a.u. over the time period $t$ $=[3000,4500]$ a.u. as the wave function evolves in the asymptotic region. The results shown in Table I are in good agreement with the QM probabilities.

\section{C. $\mathrm{H}+\mathrm{H}_{2}$ reaction}

In this section the formalism of Sec. II B is applied to the hydrogen exchange reaction $\mathrm{H}+\mathrm{H}_{2}$. The system is anharmonic and exhibits large quantum effects presenting a major challenge for semiclassical methods. The AQP method is used to propagate a wave packet, initially defined as a Gaussian in the translational coordinate $y$ and the ground rovibrational state of the diatomic. There is no overall rotation, $J=0$. Time evolution is described in the Jacobi coordinates $(x, y, \theta)$ on the Liu-Siegbahn-Truhlar-Horowitz (LSTH) potential energy surface. ${ }^{21-23}$ Equations of motion and details of the quantum trajectory implementation in arbitrary coordinates are given in Ref. 24. The quantum potential is computed from the expansion of the nonclassical momentum $\mathbf{r}$ in a basis $\{1, x, \eta(x), y\}$. The function $\eta(x)=\exp (-z x)$ determined by the Morse oscillatorlike asymptotic potential is introduced to obtain accurate asymptotic motion in the vibrational coordinate $x$ of reactants. ${ }^{24}$

For the wave function analysis the reactive part of $\psi(t)$ is transformed to the Jacobi coordinates of the products and projected on to the vibrational eigenstates of the nonrotating hydrogen molecule. We use Eq. (33) to compute elements of T projecting $\psi(t)$ onto the Gauss-Hermite functions, and then transforming $\mathbf{T}$ according to Eq. (34) to obtain the product vibrational distribution $P_{n}$. A strict definition should include projections onto rovibrational states,

$$
P_{n}=\sum_{j} \int P_{n j}(E) d E,
$$

but we neglect with the vibrational eigenfunction dependence on rotational quantum number $j$. QM vibrational distribution is obtained using the correlation function formulation of the $S$ matrix ${ }^{16}$ computed under the same assumption about the product eigenstates.

The initial wave packet in translation is a Gaussian of the form given by Eq. (16) with parameters $x_{0}=6.5$ and $\alpha$ $=4.0$. Values of the initial translational momentum $p_{0}=6$ and $p_{0}=12$ correspond to the translational energies of 0.4 and $1.6 \mathrm{eV}$, respectively. For $E=0.4 \mathrm{eV}$ total wave packet probabilities $-P=0.12$ for $\mathrm{QM}$ propagation and $P=0.11$ for AQP propagation-agree quite well. The vibrational distribution analysis reveals discrepancies between semiclassical and quantum dynamics on a finer scale. Semiclassical pro- 

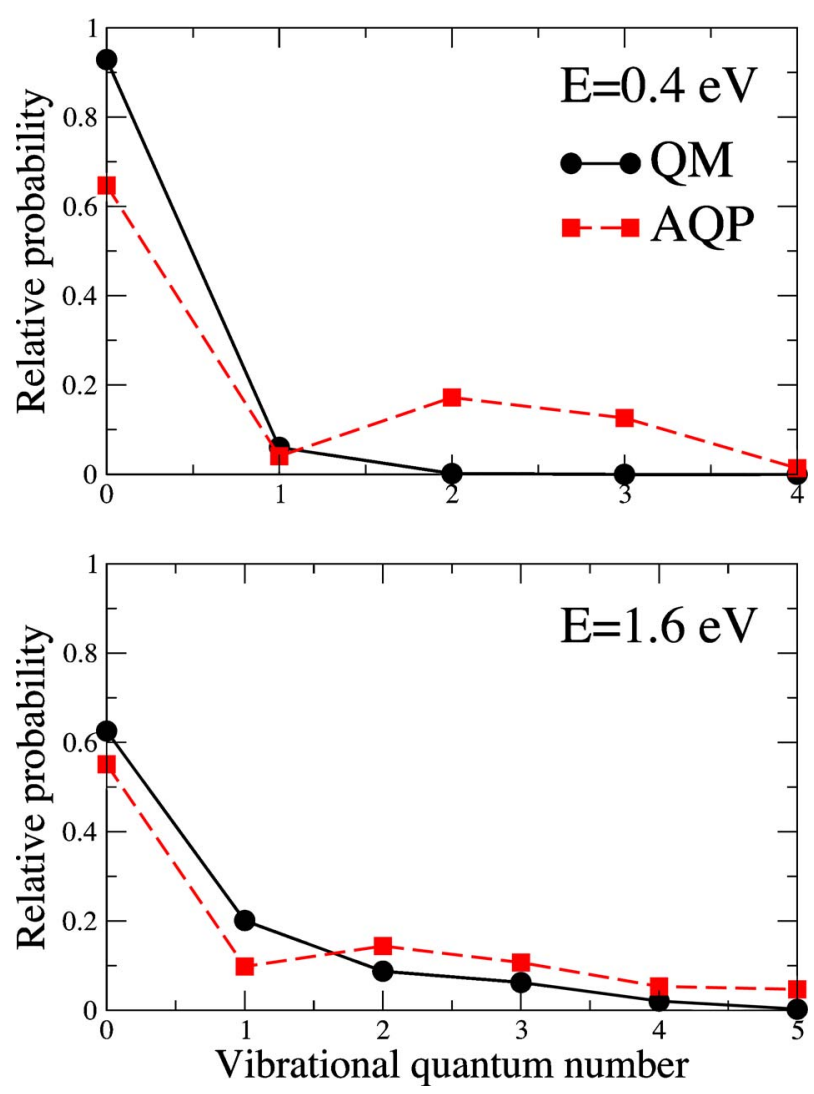

FIG. 4. (Color online) Relative final vibrational state distribution for $\mathrm{H}+\mathrm{H}_{2}$ reaction for initial translational energies $0.4 \mathrm{eV}$ (top) and $1.6 \mathrm{eV}$ (bottom). Legends are the same on both panels.

jections show a slower falloff with the quantum number than QM projections. As a result the semiclassical probabilities summed over five lowest states is one-third lower than the total wave packet probability, whereas in QM calculation vibrational states above $n=3$ are unpopulated. Calculations for $E=1.6 \mathrm{eV}$ show the same level of agreement: the wave packet reaction probabilities for a single channel are 0.35 and 0.33 for the QM and AQP propagations. The sum of semiclassical probabilities over the lowest five vibrational states that have QM population above 0.001 is 0.21 . The relative semiclassical probabilities for $1.6 \mathrm{eV}$ are in better agreement than those for $0.4 \mathrm{eV}$ as expected. The relative probabilities, $P_{n}$ for $n=0,1, \ldots$ normalized by their sum, are shown on Fig. 4. Semiclassical projection coefficients in the Gauss-Hermite and on the vibrational bases as functions of time are shown on Fig. 5. They exhibit oscillations in time on the order of $3 \%$ for the ground state and on the order of $7 \%-10 \%$ for the excited states. The probabilities were averaged over the time interval $t=[4000,5000]$ a.u. Despite significant absolute errors in the vibrational distributions the accuracy of the relative distributions improves when going from lower to higher translational energy. The total wave packet reaction probabilities agree with the QM results quite well.

\section{CONCLUSIONS}

We have presented an efficient way of computing correlation functions and wave function projections in the context

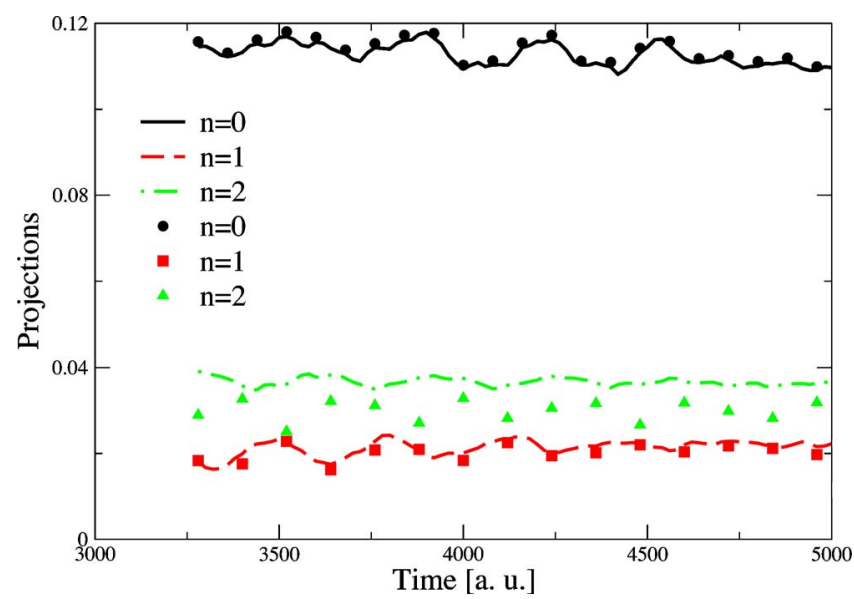

FIG. 5. (Color online) Semiclassical vibrational projection for $\mathrm{H}+\mathrm{H}_{2}$ reaction. Projections on to the Gauss-Hermite basis, $t_{n n}$, are shown as lines; projections on to the Morse oscillator eigenstates, $\widetilde{t}_{n n}$, are shown as symbols.

of quantum trajectory dynamics with the approximate quantum potential. The method scales linearly with the number of trajectories. Unlike previous approaches where the error depends directly on the fitting or interpolation error of the wave function, in the present formulation one of the two integrals over trajectories is performed exactly and the other integral is performed analytically for the second order expansion in the exponent of the integrand. This treatment of the complex integrands is often used in semiclassical methods. For example, it is the basis for the linearization procedure in the semiclassical initial value representation method described in Ref. 11. In the semiclassical limit the integrands are oscillatory and contributions to the integrals come from the lowest expansions terms. The integration is exact for Gaussian wave functions. The approximations made to the integrand are the same as have been made in the AQP propagation method. However, in the current implementation approximations to correlation functions and wave function projections are unrelated to the accuracy of the quantum trajectory propagation, which can be, in principle, exact. Therefore, the approach can be used with other exact or approximate trajectory methods that conserve probability density and is expected to be accurate for semiclassical systems.

\section{ACKNOWLEDGMENTS}

The author is grateful to Vitaly Rassolov for illuminating discussions. Production of this material was funded in part by MDA under Cooperative Agreement HQ0006-05-2-0001. Partial support from the NSF Award No. CHE-0516889 is also acknowledged.

\section{APPENDIX A: WIGNER TRANSFORMS OF THE GAUSS-HERMITE FUNCTIONS}

Compact analytical expressions are known for the matrix of the Wigner transforms ${ }^{25}$ of the Gauss-Hermite functions defined by Eq. (26) that are the eigenstates of the harmonic oscillator,

$$
\hat{H}=-\frac{1}{2 m} \frac{\partial^{2}}{\partial x^{2}}+\frac{w^{2}}{2} x^{2},
$$




$$
k=\sqrt{w \sqrt{m}}=\sqrt{2 \alpha} .
$$

With the definitions

$$
z=\frac{1}{\sqrt{2}}\left(k x+\frac{i p}{k}\right), \quad h=\frac{k^{2} x^{2}}{2}+\frac{p^{2}}{2 k^{2}},
$$

for $r^{\prime}=0$, Eq. (25) becomes

$$
\omega_{n j}=2(-1)^{n} \sqrt{\frac{n !}{j !}}(2 z)^{j-n} L_{n}^{j-n}(4 h) e^{-2 h}, \quad j \geqslant n .
$$

$L_{n}^{j-n}(x)$ are the associated Laguerre polynomials. ${ }^{13}$

\section{APPENDIX B: VIBRATIONAL PROJECTIONS FROM THE WAVE PACKET CORRELATION FUNCTIONS}

In order to obtain QM vibrational projections we compute correlation functions,

$$
C_{n}(t)=\left\langle\chi_{n} \times g \mid \psi(t)\right\rangle
$$

of $\psi(t)$ with the stationary wave packets that are direct products of the vibrational eigenstates and a Gaussian function $g(R)$ in the translational coordinate. Fourier transform of $C_{n}(t)$ normalized by the translational energy distribution of $g(R)$ gives projections

$$
t_{n}(E)=\int C_{n}(t) e^{i E t} d t
$$

Here $E$ is the total energy of the system. The probability for $\psi$ to be in the $n$th vibrational state is

$$
P_{n}=\int\left|\frac{t_{n}(E)}{\eta_{n}(E)}\right|^{2} d E
$$

where $\eta_{n}(E)$ is defined as in Ref. 16,

$$
\eta_{n}(E)=\sqrt{2 \pi}\left\langle\zeta_{n}(E) \mid g\right\rangle
$$

The translational function $\zeta_{n}(E)$ is the energy eigenstate with outgoing boundary condition corresponding to the translational energy $E-E_{n}$. $E_{n}$ is the energy of the $n$th vibrational eigenstate. For $g(R)=\phi(R, 0)$ of Eq. (16),

$$
\left|\left\langle\zeta_{n}(E) \mid g\right\rangle\right|^{2}=\frac{M}{p} \sqrt{\frac{2 \pi}{\alpha}} \exp \left(-\frac{\left(p-p_{0}\right)^{2}}{2 \alpha}\right),
$$

where $M$ is the mass in the translational degree of freedom and $p$ is the translational momentum,

$$
p=\sqrt{2 M\left(E-E_{n}\right)} \text {. }
$$

${ }^{1}$ D. Bohm, Phys. Rev. 85, 166 (1952).

${ }^{2}$ S. Garashchuk and V. A. Rassolov, J. Chem. Phys. 118, 2482 (2003).

${ }^{3}$ S. Garashchuk and V. A. Rassolov, J. Chem. Phys. 120, 1181 (2004).

${ }^{4}$ C. L. Lopreore and R. E. Wyatt, Phys. Rev. Lett. 82, 5190 (1999).

${ }^{5}$ C. J. Trahan, K. Hughes, and R. E. Wyatt, J. Chem. Phys. 118, 9911 (2003).

${ }^{6}$ R. E. Wyatt and E. R. Bittner, J. Chem. Phys. 113, 8898 (2000).

${ }^{7}$ B. K. Kendrick, J. Chem. Phys. 119, 5805 (2003).

${ }^{8}$ Y. Zhao and N. Makri, J. Chem. Phys. 119, 60 (2003).

${ }^{9}$ D. Babyuk and R. E. Wyatt, J. Chem. Phys. 121, 9230 (2004).

${ }^{10}$ E. P. Wigner, Phys. Rev. 40, 749 (1932).

${ }^{11}$ X. Sun, H. B. Wang, and W. H. Miller, J. Chem. Phys. 109, 7064 (1998).

${ }^{12}$ S. Garashchuk and V. A. Rassolov, Chem. Phys. Lett. 376, 358 (2003).

${ }^{13}$ G. Arfken, Mathematical Methods for Physicists, 3rd ed. (Academic, New York, 1985).

${ }^{14}$ E. R. Bittner, J. Chem. Phys. 119, 1358 (2003).

${ }^{15}$ M. Pulvirenti, J. Math. Phys. 47, 052103 (2006).

${ }^{16}$ D. J. Tannor and D. E. Weeks, J. Chem. Phys. 98, 3884 (1993).

${ }^{17}$ C. Leforestier, R. H. Bisselling, C. Cerjan et al., J. Comput. Phys. 94, 59 (1991).

${ }^{18}$ V. A. Rassolov and S. Garashchuk, J. Chem. Phys. 120, 6815 (2004).

${ }^{19}$ J. A. Beswick and J. Jortner, Chem. Phys. 24, 1 (1977).

${ }^{20}$ R. C. Brown and E. J. Heller, J. Chem. Phys. 75, 186 (1981).

${ }^{21}$ P. Siegbahn and B. Liu, J. Chem. Phys. 68, 2457 (1978).

${ }^{22}$ D. G. Truhlar and C. J. Horowitz, J. Chem. Phys. 68, 2466 (1978).

${ }^{23}$ W. Kolos and L. Wolniewicz, J. Chem. Phys. 43, 2429 (1965).

${ }^{24}$ V. A. Rassolov, S. Garashchuk, and G. C. Schatz, J. Phys. Chem. A 110, 5530 (2006).

${ }^{25}$ J. P. Dahl, Phys. Scr. 25, 499 (1982). 